\title{
Reanalysis of the electrode polarization in electrolytic cells limited by blocking electrodes
}

\author{
J. Ross Macdonald ${ }^{1}$ and G. Barbero ${ }^{2,3}$ \\ ${ }^{1}$ Department of Physics and Astronomy, University of North Carolina, Chapel Hill, North Carolina 27599, USA \\ ${ }^{2}$ Dipartimento di Scienza Applicata del Politecnico, Corso Duca degli Abruzzi 24, 10129 Torino, Italy \\ ${ }^{5}$ National Research Nuclear University MEPhI (Moscow Engineering Physics Institute), Kashirskoye shosse 31, \\ 115409 Moscow, Russian Federation
}

(Received 24 August 2016; published 24 October 2016)

\begin{abstract}
We evaluate the effect of ions on the electric response of an insulting liquid by means of the total electric polarization induced in a cell by an external field. The limiting surfaces are assumed blocking and identical and the ions pointike nonpolarizable charged particles. The analysis is limited to the case where the selective ionic adsorption is absent, in such a manner that in the absence of external electric field the sample is locally and globally neutral. We obtain formulas for the effective dielectric constant renormalized by the presence of the ions in the absence and presence of adsorption from the surfaces. Our results coincide with those obtained by means of the electric impedance of the cell. From the coincidence of the results relevant to the effective dielectric constant we infer that the ions in an insulating liquid do not have a conductive or dielectric nature. They are just electric charges dissolved in an insulating liquid.
\end{abstract}

DOI: 10.1103/PhysRevE.94.042608

\section{INTRODUCTION}

The influence of ions dissolved in an insulating liquid on the electric response of an electrolytic cell limited by blocking electrodes to an eternal electric field has been analyzed theoretically long ago by Macdonald [1]. The model used for the mathematical description was based on the equations of continuity for the positive and negative ions, and on the equation of Poisson for the electric potential across the cell, relating the actual potential to the bulk density of ions. This model is known as the Poisson-Nernst-Planck model (PNP). It has been generalized to take into account the effect of real electrodes on the response of the cell, and to other media in a series of papers by Macdonald and co-workers [2]. In the PNP model the liquid in which the ions are dissolved is considered as a continuum homogeneous medium, characterized by a dielectric constant (of the liquid free of ions) and by the diffusion constants for the positive and negative ions. Their influence on the electric response is related to variation of the ionic bulk density distribution. These parameters are assumed frequency independent in the range where the influence of the ions on the electric response of the cell is important, and coinciding with those in the steady state. Moreover, the ions are considered pointlike and nonpolarizable. In this framework, assuming that the external electric field is a simple harmonic function of time of small amplitude, solving the linear partial differential equations of the PNP model the electric impedance of the cell can be evaluated. The PNP model developed by Macdonald and co-workers [2] has been widely used for the dielectric characterization of liquid containing ions. The limits of the PNP model in the analysis of the experimental data have been discussed in [3-7]. According to these authors the disagreement between theoretical predictions of the PNP model and the experimental data should be expected because the original model has been developed for dilute electrolytes. Empirical correction methods which yields ion experimental data in reasonable agreement with the PNP model are proposed in [3,7].

Our aim is to evaluate the total electric polarization induced by an external electric field in a sample, in the shape of a slab, of an insulating liquid containing ions. We assume that the limiting surfaces are blocking and identical and the selective ionic adsorption neglected, in such a manner that in the absence of external electric field the sample is locally and globally neutral. We show that the effective dielectric constant of the sample determined via the electric polarization coincides with that determined via the electric impedance of the cell. Expressions for the effective dielectric constant in the presence or absence of adsorption are derived. From these results it follows that the recent model proposed by Sawada [8] is questionable.

Our paper is organized as follows. In Sec. II a general discussion on the electric polarization due to a distribution of pointlike particles is reported. The simple case in which the electrodes are not adsorbing is presented in Sec. III. In that section we show that the effective dielectric constant determined via the polarization of the cell, as for insulating media, and via the electric impedance of the cell, as for conduction media, coincide. The same type of calculation when the electrodes are adsorbing is presented in Sec. IV. Also in this case we show that the effective dielectric constant determined via polarization or via impedance coincide. Section V is devoted to the conclusions.

\section{POLARIZATION AND EFFECTIVE DIELECTRIC CONSTANT}

Let us consider a body, of volume $\tau$ limited by a surface $\Sigma$, containing a charge distribution described, in the continuum approximation, by the charge density $\rho(\mathbf{r}, t)$. The time dependence of the charge distribution $\rho(\mathbf{r}, t)$ can be due to the motion of the charges inside the body. We assume that the body is globally neutral, and hence

$$
\int_{\tau} \rho(\mathbf{r}, t) d \tau=0 .
$$

In this framework the dipole moment is independent of the origin of the reference frame used for the description, and 
given by [9]

$$
\mathbf{p}(t)=\int_{\tau} \mathbf{r} \rho(\mathbf{r}, t) d \tau .
$$

In the case where the sample has the shape of a slab of thickness $d$ and surface area $S$, we use a Cartesian reference frame having the $z$ axis perpendicular to the limiting surfaces, at $z \pm d / 2$, and $\rho(\mathbf{r}, t)=\rho(z, t)$ only. In this situation the dipole moment of the body is parallel to the $z$ axis $\mathbf{p}(t)=p(t) \mathbf{u}_{\mathbf{z}}$ where $\mathbf{u}_{\mathbf{z}}$ is the unit vector parallel to the $z$ axis, and $p(t)$ is given by

$$
p(t)=S \int_{-d / 2}^{d / 2} z \rho(z, t) d z .
$$

In the case where the charge is partially distributed in the bulk, with a bulk density $\rho_{b}(z, t)$ and partially on the surfaces, with a surface density $\pm \sigma(t)$, the charge density is given by

$$
\rho(z, t)=\rho_{b}(z, t)+\sigma(t) \delta(z-d / 2)-\sigma(t) \delta(z+d / 2),
$$

where $\delta$ is Dirac's function. For the charge distribution given by (4) the dipole moment is

$$
p(t)=S\left\{\int_{-d / 2}^{d / 2} z \rho_{b}(z, t) d z+\sigma(t) d\right\} .
$$

The polarization, $\mathbf{P}(t)$, is defined as the bulk density of the dipole moment, $\mathbf{P}(t)=d \mathbf{p} / d \tau$, and its spatial average value is $\langle\mathbf{P}(t)\rangle=\mathbf{p}(t) / \tau$. The average polarization of our body in the shape of a slab is then along the $z$ axis of amplitude,

$$
\langle P(t)\rangle=\frac{1}{d} \int_{-d / 2}^{d / 2} z \rho_{b}(z, t) d z+\sigma(t) .
$$

If the charge distributions described by $\rho_{b}(z, t)$ and $\sigma(t)$ are due to the application of an external difference of potential $\Delta V(t)$ to the electrodes in such a manner that the electrodes are at $V( \pm d / 2)= \pm V / 2$ it is possible to define an effective dielectric constant of the body by means of the macroscopic relation

$$
\langle\mathbf{P}(t)\rangle=\left[\varepsilon_{\mathrm{eff}}-\varepsilon(0)\right] \mathbf{E}_{\mathrm{ext}}(t),
$$

where $\mathbf{E}_{\text {ext }}(t)=-[V(t) / d] \mathbf{u}_{\mathbf{z}}$ is the external field applied to the sample, and $\varepsilon(0)$ is the dielectric constant of the medium in the absence of the external field. For the case under investigation where the sample is in the shape of a slab, we get

$$
\varepsilon_{\mathrm{eff}}=\varepsilon(0)-\frac{d}{V(t)}\left\{\frac{1}{d} \int_{-d / 2}^{d / 2} z \rho_{b}(z) d z+\sigma\right\} .
$$

The analysis presented above is valid in the quasistatic limit when the continuum description of the electric charge works well. This means that the electric charges are pointlike and are distributed in a continuous manner in the insulating medium of dielectric constant $\varepsilon(0)$. In this framework Eq. (8) gives the effective dielectric constant of the medium containing the electric charges. We note that in the analysis the charges are supposed pointlike, and nonpolarizable. Hence the presence of the charges does not modify the value of $\varepsilon(0)[10,11]$. The case where the ions are polarizable particles, discussed in [12], is more complicated. In this case the ions also give a contribution to the dielectric spectrum. However in our analysis, as in that reported in [8], the small contribution to the effective dielectric constant related to the polarizability of the ions is not taken into account. To generalize our analysis to this more realistic situation, it is necessary to evaluate the contribution to the effective dielectric constant related to the ions redistribution, not only related to their electric charge, but also to their polarizability. This can be done considering the solution as a mixture, along the line discussed in [9] (p. 65). The work is in progress.

We can now apply the formalism presented above, well known in the static cases, to the situation in which the charge is due to ions dispersed in an insulating liquid. We consider a liquid containing, in thermodynamical equilibrium, a bulk density of ions $N_{0}$, very small with respect to the bulk density of the liquid $N$. In this case the assumption of full dissociation works well, and the generation-recombination phenomenon can be neglected. The ions are assumed identical in all aspects, except for the sign of the electrical charge, indicated by $q$. In particular they have the same diffusion coefficients $D$ and electric mobilities $\mu$. Also the electrodes are assumed identical with respect to the ions. This implies that there is not selective adsorption from the electrodes. In the presence of an external difference of potential $V(t)=V_{0} \exp (i \omega t)$, the ions move toward the electrode of opposite sign. We indicate by $n_{p}(z, t)$ and $n_{m}(z, t)$ the bulk density of positive and negative ions in the sample in the presence of the external field, and by $s_{p}(d / 2, t), s_{m}(d / 2, t)$ and $s_{p}(-d / 2, t), s_{m}(-d / 2, t)$ the surface density of positive and negative ions at $z= \pm d / 2$, respectively. For the symmetry of the problem $n_{p}(z, t)=-n_{m}(-z, t)$, as well as $s_{p}(d / 2, t)=-s_{m}(-d / 2, t)=s(t)$ and $s_{m}(d / 2, t)=$ $-s_{p}(-d / 2, t)=-s(t)$.

\section{NO ADSORPTION FROM THE ELECTRODES}

Let us consider first that case where the electrodes are not adsorbing, and hence $s(t)=0$. Indicating by $\varepsilon_{b}$ the dielectric constant of the liquid free of ions, we have $\varepsilon(0)=\varepsilon_{b}$. In this framework, as discussed elsewhere [13], for small $V_{0}$,

$$
\left.n_{(} z, t\right)=-n_{m}(z, t)=p_{0} \sinh (\beta z) \exp (i \omega t),
$$

where

$$
\beta=(1 / \lambda) \sqrt{1+i\left(\omega / \omega_{D}\right)},
$$

and

$$
p_{0}=-\frac{\varepsilon_{b} \beta^{2}}{4 q} \frac{V_{0}}{\sinh (\beta d / 2)+i\left(\omega / \omega_{D}\right)(\beta d / 2) \cosh (\beta d / 2)} .
$$

In (10) $\lambda$ is the length of Debye defined by $\lambda=$ $\sqrt{\varepsilon_{b} K_{B} T /\left(2 N_{0} q^{2}\right)}, K_{B} T$ is the thermal energy, and $\omega_{D}=$ $D / \lambda^{2}$ the circular frequency of Debye. The bulk charge density is

$$
\rho(z, t)=q\left[n_{p}(z, t)-n_{m}(z, t)\right]=2 p_{0} \sinh (\beta z) e^{i \omega t},
$$

and the total dipole moment of the cell, defined by (3) and taking into account (11), is then

$$
p(t)=-\varepsilon_{b} V_{0} S \frac{\beta d / 2-\tanh (\beta d / 2)}{\tanh (\beta d / 2)+i\left(\omega / \omega_{D}\right) \beta d / 2} e^{i \omega t} .
$$


It follows that the spatial average polarization $\langle P(z, t)\rangle=$ $p(t) /(S d)$, is found to be

$$
\begin{aligned}
\langle P(z, t)\rangle= & -\varepsilon_{b} \frac{V_{0}}{d} \frac{1}{\lambda^{2} \beta} \\
& \times \frac{\beta d / 2-\tan (\beta d / 2)}{\left(1 / \lambda^{2} \beta\right) \tanh (\beta d / 2)+i(\omega d / 2 D)} e^{i \omega t} .
\end{aligned}
$$

Since in the present case

$$
\langle P(z, t)\rangle=\left(\varepsilon_{\mathrm{eff}}-\varepsilon_{b}\right) E_{\mathrm{ext}}(t)=-\left(\varepsilon_{\mathrm{eff}}-\varepsilon_{b}\right) \frac{V_{0}}{d} e^{i \omega t},
$$

we derive for $\varepsilon_{\text {eff }}$ the final expression

$$
\varepsilon_{\mathrm{eff}}=\varepsilon_{b}\left\{1+\frac{\beta d / 2-\tanh (\beta d / 2)}{\tanh (\beta d / 2)+i\left(\omega / \omega_{D}\right) \beta d / 2}\right\} .
$$

From Eq. (16) the real $\varepsilon_{\text {eff }}^{\prime}$ and imaginary $\varepsilon_{\text {eff }}^{\prime \prime}$ of the effective dielectric constant can be easily obtained. A numerical analysis of this problem, along the line discussed above, has been reported by Sawada a few years ago [14].

In [13] by means of the PNP model has been evaluated, with the same simplifying hypotheses of one group of ions with identical ions in a sample limited by blocking electrode, the electric impedance of a cell $Z$ in the shape of a slab. Its expression is

$$
Z=-i \frac{2}{\omega \varepsilon_{b} \beta^{2} S}\left\{\frac{1}{\lambda^{2} \beta} \tanh (\beta d / 2)+i \frac{\omega d}{2 D}\right\} .
$$

Using the concept of complex impedance, $\mathcal{C}=\epsilon S / d$, where $\epsilon$ is the effective complex dielectric constant determined by means of the impedance, assuming the ions as moving in a conductive medium, we have $Z=-i /(\omega \mathcal{C})$, from which

$$
\epsilon=-\frac{i}{\omega Z} \frac{d}{S}
$$

Substituting (17) into (18) we get for $\epsilon$ the expression

$$
\epsilon=\varepsilon_{b} \beta d / 2 \frac{1+i\left(\omega / \omega_{D}\right)}{\tanh (\beta d / 2)+\left(\omega / \omega_{D}\right)(\beta d / 2)},
$$

that coincides with (16), i.e., $\epsilon=\varepsilon_{\text {eff }}$ [7].

The conclusion of this simple calculation is that the ionic charges have neither a dielectric nor conductive characteristics. They are just electric charges, whose motion and influence on the electric potential is described by the equation of continuity and the equation of Poisson, relating the bulk density of ions to the actual electric potential. Their influence on the electric response of a cell to an external stimulus can be evaluated by means of the average polarization, as for dielectric media, or by means of the electric impedance, as for conductive media. The results coincide for all frequencies.

We stress that the external charges sent by the power supply on the electrodes have not to be taken into account in the calculation of the polarization induced by the field on the medium. Of course if we are interested in the total dipole moment of the cell with respect to points outside of the cell, the dipole moment expression is $p_{\text {tot }}(t)=p(t)+\sigma_{\text {ext }}(t) S d$, where $\sigma_{\text {ext }}(t)$ is the external charge sent by the power supply to fix the difference of potential between the electrodes. But this total dipole moment has not any interest in the analysis of the response of the cell to an external stimulus. If this quantity is considered important, it can be evaluated in the following manner.

From Poisson's equation $\boldsymbol{\nabla} \cdot \mathbf{D}=\rho$, for our one dimensional problem, we obtain $d E / d z=\rho / \varepsilon_{b}$, and hence

$$
E(z, t)=E(-d / 2, t)+\frac{1}{\varepsilon_{b}} \int_{-d / 2}^{z} \rho(x, t) d x .
$$

In the case under consideration, where $V( \pm d / 2, t)=$ $\pm\left(V_{0} / 2\right) \exp (i \omega t)$, simple considerations show that $E(-d / 2, t)=-\sigma_{\text {ext }}(t) / \varepsilon_{b}$. Consequently from (20) we obtain

$$
\sigma_{\mathrm{ext}}(t)=-\varepsilon_{b} E(z, t)+\int_{-d / 2}^{z} \rho(x, t) d x .
$$

Integrating over the thickness of the sample we easily get

$$
\sigma_{\mathrm{ext}}=\frac{1}{d}\left\{\varepsilon_{b} V_{0}+\int_{-d / 2}^{d / 2}\left(\int_{-d / 2}^{z} \rho(x, t) d x\right) d z\right\} .
$$

Taking into account (9) and (11) we finally obtain

$$
\sigma_{\mathrm{ext}}(t)=\varepsilon_{b} \frac{V_{0}}{d}\left\{1+\frac{\beta d / 2-\tanh (\beta d / 2)}{\tanh (\beta d / 2)+i\left(\omega / \omega_{D}\right) \beta d / 2}\right\} e^{i \omega t} .
$$

Note that in the framework of the present calculus, where the ionic charges remain in the bulk, the total dipole moment of the cell, due to the internal and external charges, is independent of the bulk density of ions, and it is given by $p_{\text {tot }}(t)=\varepsilon_{b} V_{0} S \exp (i \omega t)$, as shown in a different manner on a particular case in [10].

\section{ADSORPTION FROM THE ELECTRODES}

Let us consider now the situation in which the ionic charges are adsorbed by the electrodes. In the framework of previous analysis (sample in the shape of slab, limited by identical electrodes, one group of positive and negative ions, identical in all aspects, except for the sign of the electric charge) in Langmuir's approximation the adsorption of positive ions at $z=d / 2$ is described by

$$
\frac{d s_{p}}{d t}=\kappa n_{p}(d / 2, t)-\frac{1}{\tau} s_{p}(t),
$$

where $n_{p}(d / 2, t)$ is the bulk density of positive ions just in front of the electrode, $\kappa$ and $\tau$ are the adsorption coefficient and desorption time, respectively [15]. For the symmetry of the problem $n_{p}(z, t)=-n_{m}(-z, t)$, as well as $s_{p}(d / 2, t)=$ $-s_{m}(-d / 2, t)=s(t)$ and $s_{m}(d / 2, t)=-s_{p}(-d / 2, t)=-s(t)$. The net surface charge density is $\sigma(d / 2)=2 q s(t)$. As before we assume that the sample is submitted to a periodic external voltage of the type $V(t)=V_{0} \exp (i \omega t)$. In this case in the steady state Eq. (9) is still valid, and

$$
s(t)=p_{0} \frac{\kappa \tau}{1+i \omega \tau} \sinh (\beta d / 2) \exp (i \omega t)
$$

where $p_{0}$ has to be determined by means of the boundary conditions related to the surface current density and to the 
electric potential, as discussed in [15]. A simple calculation gives

$$
p_{0}=-\frac{\varepsilon_{b} \beta^{2}}{4 q} \frac{V_{0}}{\left[1+\left(\omega / \omega_{D}\right) \mathcal{H}(\omega)\right] \sinh (\beta d / 2)+i\left(\omega / \omega_{D}\right)(\beta d / 2) \cosh (\beta d / 2)},
$$

where

$$
\mathcal{H}(\omega)=\frac{\kappa \tau d}{2 \lambda^{2}} \frac{1+i \omega / \omega_{D}}{1+i \omega \tau}
$$

is a complex number frequency dependent taking into account the adsorption phenomenon. Note that in the absence of adsorption ( $\kappa=0$ and $\tau=0$ ), $\mathcal{H}$ vanishes identically, and Eq. (26) coincides with Eq. (11), as expected.

In this case, repeating the calculation of the previous section, starting from Eq. (8), deduced for insulating media, we get for the effective dielectric constant of the sample the expression

$$
\varepsilon_{\mathrm{eff}}=\varepsilon_{b}\left\{1+\frac{\beta d / 2-[1-\mathcal{H}(\omega)] \tanh (\beta d / 2)}{\left[1+i\left(\omega / \omega_{D}\right) \mathcal{H}(\omega)\right] \tanh (\beta d / 2)+i\left(\omega / \omega_{D}\right) \beta d / 2}\right\},
$$

that generalizes Eq. (16) to take into account the adsorption phenomenon, in Langmuir's approximation. From this expression the frequency dependencies of the effective real $\varepsilon_{\text {eff }}^{\prime}$ and imaginary $\varepsilon_{\text {eff }}^{\prime \prime}$ parts of $\varepsilon_{\text {eff }}$ can be derived.

The electric impedance of the cell in the presence of adsorption has been obtained in [15] and can be written as

$$
Z=-i \frac{2}{\omega \varepsilon_{b} \beta^{2} S[1+\mathcal{K}(\omega)]}\left\{\frac{1}{\lambda^{2} \beta} \tanh (\beta d / 2)+i[1+\mathcal{K}(\omega)] \frac{\omega d}{2 D}\right\},
$$

where

$$
\mathcal{K}(\omega)=\frac{\beta \kappa \tau}{1+i \omega \tau} \tanh (\beta d / 2),
$$

is another complex number frequency dependent taking into account the adsorption phenomenon. In the absence of adsorption $(\kappa=0$ and $\tau=0), \mathcal{K}=0$ and Eq. (30) coincides with Eq. (17), as expected. Substituting (30) into (18) we obtain for the effective complex dielectric constant, determined via the impedance, the expression

$$
\epsilon=\varepsilon(\beta d / 2) \frac{\left[1+i\left(\omega / \omega_{D}\right)\right][1+\mathcal{K}(\omega)]}{\tanh (\beta d / 2)+i\left(\omega / \omega_{D}\right)[1+\mathcal{K}(\omega)]},
$$

that coincides, identically, with (28).

\section{CONCLUSIONS}

We have analyzed the influence of the ions on the effective dielectric constant of a dielectric medium. The analysis has been limited to the case in which the sample is in the shape of a slab, and only one group of positive and negative ions is present in the liquid. We assumed furthermore that positive and negative ions are identical in all aspects, except of the sign of the electric charge. The effective complex dielectric constant has been determined via the electric polarization of the cell, as in insulating media, taking into account the ionic distribution. We have also determined the effective dielectric constant via the electric impedance of the cell, as in conducting material, taking into account the effective total electric current across the cell, due to the conduction and to the displacement. We have shown that the dielectric constants determined by means of the two methods are identical. From this result it follows that the ions in an insulating liquid do not have conductive or dielectric nature, as suggested recently [8]. They are pointlike particles, whose description is based on the continuity equation, stating the conservation of particles, and on the equation of Poisson relating the effective electric potential across the cell with the charge density.

\section{ACKNOWLEDGMENT}

This work was partially supported by the Competitiveness Program of National Research Nuclear University (NRNU) MEPhI (Moscow Engineering Physical Institute).
[1] J. R. Macdonald, Phys. Rev. 92, 4 (1953).

[2] All the works of Macdonald are accessible, in pdf format, at http://jrossmacdonald.com.

[3] Y. Wang, C.-N. Sun, F. Fan, J. R. Sangoro, M. B. Berman, S. G. Greenbaum, T. A. Zawodzinski, and A. P. Sokolov, Phys. Rev. E 87, 042308 (2013).

[4] M. Z. Bazant, K. Thornton, and A. Ajdari, Phys. Rev. E 70, 021506 (2004).

[5] M. Z. Bazant, K. T. Chu, and B. J. Bayly, SIAM J. Appl. Math. 65, 1463 (2005).

[6] A. Serghei, M. Tress, J. R. Sangoro, and F. Kremer, Phys. Rev. B 80, 184301 (2009).

[7] R. J. Kortschot, A. P. Philipse, and B. H. Herné, J. Phys. Chem. C 118, 11584 (2014).
[8] A. Sawada, Phys. Rev. E 93, 052608 (2016).

[9] L. Landau and E. Lifchitz, Electrodynamique des Milieux Continus (MIR, Moscow, 1990).

[10] G. Barbero and I. Lelidis, J. Appl. Phys. 115, 194101 (2014).

[11] A. L. Alexe-Ionescu, G. Barbero, and I. Lelidis, J. Chem. Phys. 141, 084505 (2014).

[12] K. F. Rinne, S. Gekle, and R. R. Netz, J. Phys. Chem. A 118, 11667 (2014).

[13] G. Barbero and A. L. Alexe-Ionescu, Liq. Crystallogr. 32, 943 (2005).

[14] A. Sawada, J. Chem. Phys. 129, 064701 (2008).

[15] G. Barbero, Phys. Rev. E 71, 062201 (2005). 